\title{
Ergonomic analysis for the assessment of the risk of work-related musculoskeletal disorder in forestry operations
}

\author{
Raimondo Gallo, Fabrizio Mazzetto \\ Free University of Bozen-Bolzano, Faculty of Science and Technology, Bolzano (BZ) Italy
}

\begin{abstract}
The risk to run into a Work-Related Musculoskeletal Disorder (WMSD) is very high when operating in the primary sector. As a matter of fact the professional illnesses related to the WMSD in Italy are increasing. Nowadays the assessment of the WMSD in the primary sector is performed mainly in the agricultural sector, considering different agronomical activities; for the forestry sector, only few documents reported an ergonomic evaluation. The lack of available information on this topic in the forestry sector, as well as the similarity with the agricultural sector, drives the interest to the assessment of the conditions that expose workers to WMSD risks in forestry operations.

Four different assessment approaches were applied in this study. These tools permitted to classify which are the exposures and if there is the presence of WMSD risk for forest operators. The approaches are respectively the OCRA checklist and the RNLE equation, both recognized as ISO standards, as well as OWAS and REBA, recommended by ISO standards. The first approach focusses on the risk due to repetitive and stressed movements, while the second evaluates the risk of injures due to the manual movement of loads. Meanwhile OWAS and REBA detect the possibility of injures due to wrong postures during the work. These approaches were applied for the cutting operations with chainsaw. Since the evaluation requires high levels of attention and also because it was necessary to gain a good level of safety for the surveyor, a digital camera was used to film the operative activities. Then, the movies were analysed in office. Aim of the research is to analyse if it is possible to apply the approaches suggested by ISO standards in order to assess the most dangerous activities that, when not properly carried out, could be the cause of WMSD in forestry operations. The study showed that during the use of the chainsaw the index of risk of professional injures was overpassed several times, compromising the operator's safety.
\end{abstract}

Correspondence: Raimondo Gallo, Free University of Bozen-Bolzano, Faculty of Science and Technology, Piazza Universitá 5, 39100 Bolzano (BZ) Italy. Email: raimondo.gallo@natec.unibz.it

Key words: physical ergonomics; biomechanical overload; exposure factors; postures assessment; risk of injures.

(C) Copyright R. Gallo and F. Mazzetto, 2013

Licensee PAGEPress, Italy

Journal of Agricultural Engineering 2013; XLIV(s2):e147

doi:10.4081/jae.2013.s2.e147

This article is distributed under the terms of the Creative Commons Attribution Noncommercial License (by-nc 3.0) which permits any noncommercial use, distribution, and reproduction in any medium, provided the original author(s) and source are credited.

\section{Introduction}

All typologies of manual work, even if with different levels, present the possibility to cause professional illnesses, constituting a risk for the operator's safety. In the specific literature these illnesses are called work-related musculoskeletal disorders - WMSD. The name WMSD refers to all disorders of joints as well as muscles due to traumas, micro-traumas repeated in the time and overload of muscles and joints (articulation) (Occhipinti, Colombini, 1996; Zanuttini, 2005). The national Institute for Occupational Safety and Health (Niosh, 1981) correlates the causes of WMSD to the heavy physical work, to the application of forces for lifting and moving loads, to the unbalanced lifting, to the bending and twisting of the articulation, to the velocity of movements and to the uncorrected postures. The assumption of a wrong posture causes the wrong coordination of the rachides' muscles, up to a failure. Other complementary factors that increment the risk of WMSD injuries are the use of equipment that transmits vibrations (Whole Body Vibration WBV and Hand Arm Vibration HAV) and the low temperature in the working place (Colombini et al. 2010, Calvo, 2009, Zanuttini, 2005). For this re ason committees that treat health topics - international as well as national - edited standards, laws and guide lines about the procedure of monitoring and assessment of risk of injures due to manual handling of loads and postures. These methods evaluate the risk of WMSD for upper limbs (Colombini et al., 2011; Colombini, Occhipinti, 2011; Colombini et al., 2007) for rachides (Colombini et al., 2010) and postures (Calvo, 2009; Zanuttini et al., 2005; Hignett 2000).

Considering all factors of work-related injuries it is possible to assume that the primary productive sector is interested by having activities which could cause the arising of professional illness. Indeed, in a national report published in the 2012 by the INAIL National Institute for the assessment of injures on work - is reported that WMSD in the primary sector increased about $620 \%$ since 2007. About $39 \%$ of those injures interest the intervertebral discs and about $26 \%$ the tendons. This is the reason for the importance to identify which are the levels of risk that determine WMSD in the operations of the primary productive sector.

Nowadays, consulting the specific literature, several documents treat the assessment of work-related musculoskeletal disorders in agriculture (Camilleri et al., 2012; Colantoni et al., 2012; Murgia et al., 2012; Schillaci et al., 2011; Cecchini et al., 2010; Pressiani et al,. 2010; Colombini et al., 2007), while only few of them refer to the forest sector (Calvo, 2009; Zanuttini 2005; Ashby, 2001). Aim of this work is to evaluate if the assessment methodologies already applied in the agricultural and in the industrial sector could be employed also in forestry. At this regard the assessment recommended by ISO 11228, EN 1005 - OCRA index, NIOSH index - and those recommended by standards - OWAS index, REBA index -, were applied for the WMSD assessment. 


\section{Materials and methods}

As consequence of the importance of the operating time relief, the present study is done in parallel to ones that analyse the application of IT equipment for time monitoring during logging operations.

The study-case was set in a forest of spruce, where cultural operations of thinning were planned. The operations were organized in the municipality of Rodenek, in the Autonomous Province of Bozen (N-E of Italy) in a forest managed by the State-Owned Forest Company. Besides the silvicultural management task, the provincial company must execute all silvicultural exploitations. The harvesting operations consisted of thinning operations.

The filming was performed with a Nikon ${ }^{\circledR}$ S8000, a 14 megapixel digital camera that permitted to record the movies in high definition. For keeping the camera in the right position a tripod was used.

The forestry operations had interested a part of forest with 3rd and 4th class of slope, this means that it presents a slope from around $40 \%$ to $80 \%$. (Hippoliti and Piegai, 2000). This morphological characteristic, associated to superficial humidity in the first hours of the day, characterized the working site to be very slippery and unstable.

The working operation of cutting and falling down, limbing (both branches and tops), cross-cutting, yarding, logging and transportation are reported in the specific literature (Hippoliti, Piegai, 2000, Cividini, 1991) as well as the sequence of forestry work. The present paper would focus its analysis on the ergonomic aspect of the cutting operations. Authors consider these operations as the most critical for the operator's safety due to the high number of movements with a load in hand - the chain saw -.The operation of cutting consists in the division of the aboveground biomass from the roots; causing a modification of the centre of gravity it is possible to change the tree's attitude from vertical to horizontal. Usually this operation is done through the use of a chain saw with cuts done with successive movements. At each movement, similar to the lever movement, every cutting tooth of the chains saw penetrates into the wood until the complete separation. Since this operation is done on the ground level, mainly on mountain steep sides, the operator is forced to assume, with a load in hand, wrong postures and also to do repetitive movements, those could be characterized by long periods of exposure (Figure 1).

\section{Detection approaches}

Checklist OCRA and RNLE by NIOSH as well as the OWAS and REBA methods were applied respectively in order to assess the index of exposure at WMSD and to identify the presence or not of risks of injuries.
The Checklist OCRA - Occupational Repetitive Actions - is a simplification of the OCRA index method. Through a rapid evaluation, the method permits to assess the presence of risks, their mapping and their first managing (Colombini et al., 2011). Aim of this evaluation is to assess the risk of biomechanical overload of operators' upper limbs. The use of the checklist OCRA requires the recognition of the technical actions, considered as the elementary actions done by the operator in order to perform a task, also the evaluation of multiple risk factors like recovery period (RP), movements frequency (Fr), use of physical forces (F) and wrong posture evaluation as well as stereotypy (Ps). Besides these, also the evaluation of the net repetitive working time (NRWT) as well as the complementary factors (CF) (vibrations, low temperatures, equipment's kickbacks, etc.) was done (Colantoni et al., 2012; Colombini et al., 2011; Colombini, Occhipinti, 2011; Colombini et al., 2007). In this case the equation that describes the Checklist OCRA index is:

Checklist OCRA = Fr x F x Ps x CF x mRP x mNRWT

(Colombini, Occhipinti, 2011)

In the equation, the RP and NRWT are reported as multiplicative factor.

Following the ISO 12295, the application of these methodologies must respect one of two criteria:

The assessed operation must be composed by work cycles;

The work must be characterized by the same repetitive technical actions for more than $50 \%$ of the working task.

The NIOSH (National Institution for Occupational Safety and Health) staff developed the RNLE - Revisited NIOSH Lifting Equation -. This equation permits the calculation of the risk of WMSD at rachides level. Thanks to this evaluation it is possible to obtain the lifting index (LI). The lifting index is "a term that provides a relative estimate of level of physical stress associated with a particular manual lifting task" (Waters et al., 2007). In order to obtain the LI, a load constant (LC) - obtained according to gender and age of operator (Colombini et al., 2010) - multiplied by factors of stress was calculated. The considered factors of stress are: the horizontal distance from load to operator (HM), the vertical height of the lift (VM), the vertical displacement during the lift (DM), the angle of asymmetry of the trunk (AM), the frequency and duration of lifting (FM), the quality of hand-object coupling (CM). With these data it is possible to calculate the recommended weight limit (WLR), as reported in the follow equation:

$$
\text { WRL }=\text { LC } \times \text { HM } \times \text { VM } \times \text { DM } \times \text { AM } \times \text { FM } \times \text { CM }
$$

(Waters, et al., 1994)

The lifting index is based on the relationship between the weight of the load (L) listed during the task and the WRL, as reported in the equation below:
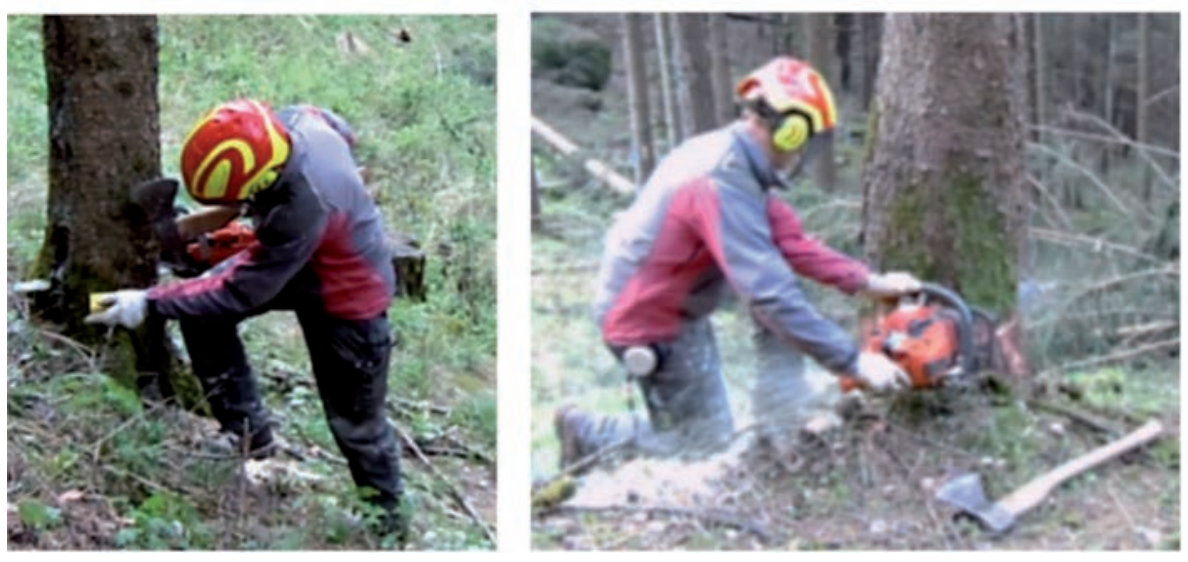

Figure 1. Example of possible wrong posture assumed by the operator during cutting operations. 


\section{$\mathrm{LI}=\mathrm{L} / \mathrm{WRL}$}

(Waters, et al., 1994)

It is very important to underline that this method is usable if the load is more than $3 \mathrm{~kg}$, or when the handling of the object happens more than once every 5 minutes (Colombini et al., 2010) and for those repetitive operations that are cadenced by an industrial machine process (Zanuttini, 2005).

In order to analyse if the posture assumed by an operator is correct or could be in risk of WMSD, it is possible to employ two detecting tools: OWAS and REBA. The OWAS - Ovako Working-posture Analysis System - observes which are the positions of back, arms, legs and also the weight of the load lifted (Li, Buckle, 1999; Zanuttini, 2005). A codification for each of the above mentioned positions was done thanks to a comparison with references reported in literature (Lundqvist, Gustafsson, 1987). So each posture is described by a code - the OWAS code - with four scores, each of them evaluates the "weight" that each posture has on the global posture. Besides using the global postural codes in a multi-entry table it is possible to calculate the class of risk $(1,2,3,4)$ for each posture (Louhevaara, Suurnäkki, 1992). Considering the number of observation for each class of risk during the relief period it is possible to calculate the frequency of observation (a, b, $c, d$ ) of a posture for each task. The index of risk is calculated from the sum of the products between frequencies and respective class of risk, as reported in the following equation:

$$
\begin{gathered}
I=[(\mathrm{a} \times 1)+(\mathrm{b} \times 2)+(\mathrm{c} \times 3)+(\mathrm{d} \times 4)] \times 100 \\
\text { (Louhevaara, Suurnäkki, 1992) }
\end{gathered}
$$

Also REBA - Rapid Entire Body Assessment - is based on the association of scores, in relation to the posture assumed by the operators. REBA method assigns a code for each posture analysing the range between the angles of effective postures, taken during the working operation, and those of neutral positions. The analysed body parts are: the trunk, the neck, the legs (Group A), the upper arms, the lower arms and the wrists (Group B). The last groups are scored separately for different sides. Beside this, the method assigns a score - positive or negative - in relation to the frequency of the movements, to the presence of loads or to the application of forces and to the hand-object coupling (Hignett, McAtamney, 2000; McAtamney, Hignett, 2005). Using multientry tables, firstly specific for the two groups relieved then for a global evaluation, it is possible to calculate the total REBA score. The methodology is not based on a real equation but on a scoring sheet.

Anyhow, Authors suggest to refer to the specific literature for a more detailed description of the methodologies mentioned above (Colombini et al., 2011; Colombini, Occhipinti, 2011; Colombini et al., 2010; Colombini et al., 2007; Li, Buckle, 1999; Zanuttini, 2005; Hignett, McAtamney, 2000; McAtamney, Hignett, 2005).

\section{Procedure followed}

The application of the cited assessment methodologies requires the direct analysis of the working operations in two different ways. Checklist OCRA and RNLE require an analysis of a period of work considered as sample. During this survey a dynamic assessment is made. So, the sample is representative of the whole working period. While OWAS and REBA, require the analysis of the singular posture assumed by the operator during his task. In this case the survey is made considering a static postural sample.

In order to obtain this observation, since the evaluation requires high levels of attention and also because it was necessary to gain a good level of safety for the surveyor, a digital camera was used to film the operative activities. Then, the movies were analysed in office in order to obtain the analysis for the different methodologies. At this regard KINOVEA, a free software for the video analyst, was used for all assessments. Indeed this software results useful both for the dynamic and for the static surveys, because it permits to make a photo sequence at fixed intervals: in our case every 5 seconds. Thanks to the KINOVEA software it was possible to measure also all joint angles for REBA assessment.

Finally, specific softwares developed by the epmresearch group of Milan (http://www.epmresearch.org) to calculate OCRA and NIOSH index and score sheets necessary to OWAS and REBA evaluation (Hignett, McAtamney, 2000, Louhevaara, Suurnäkki, 1992), were used in order to assess the level of risk or to identify it.

\section{Results and discussions}

The ergonomic assessment was done in parallel with a study of time monitoring for the application of systems for the automatic monitoring relief. Since the Authors wanted to evaluate the feasibility of the application of several methodologies for the ergonomic assessment, a sample period of half an hour of movie was taken. In this sample several operations of cutting were recorded. In office this movie was divided in movies, as many as the number of cutting operations. The Authors, in this context, consider that the cutting phase starts when the operator picks up the chain saw in order to use it and finishes when the cut is done and the operator turns it off.

Although the different methodologies require the analysis of different aspects, it was possible to use only one movie since it satisfied all ergonomic methodologies requirements. In the other movies, even if the positioning of the camera was done with care - considering felling direction, operator position - the trunk as well as the operator's body position did not permit a good and easy observation of the upper limbs movements and also the operator's postures. So the representative movie sample amounts to 87 second of recording on which OCRA and NIOSH index were analysed and 18 frames (5 second for each one) on which OWAS and REBA assessment were done.

As far as the assessment of the two indexes is concerned, a critical situation was found in both.

The application of the OCRA checklist approach is possible because the operations are characterized by repeated movements for more than half of the time of observation (Colombini et al., 2010). Considering the OCRA index, from the analysis of the video, it was possible to recognize 44 and 19 technical actions respectively for the right and left upper limb (Table 1).

In this case the operator performed operative actions with a frequency of 30.3 actions/minute whit the right hand and 13.1 actions/minute with left hand. In Table 2 the summary of the assessment done through OCRA checklist approach is reported. According to Table 2, the operation of cutting presented two different values of OCRA checklist index.

Besides the numerical index, the classification by colours permits to easily identify the presence of criticism. Therefore the result of the assessment underlines the presence of a medium and high risk of WMSD for right and left limb respectively (values between $14.1-22.5$, red colour; values $\geq 26.6$, purple colour). This difference of the risk of injures is mainly due to the wrong postures that the woodman assumed during the cutting operations. Among all scores, the biggest role on the assessment is played by the long period in which the operator keeps the left arm rotated, in order to maintain a good grip on the handle of the chain saw, which affects the elbow. The use of this approach, indirectly, permits also the identification of the actions that are necessary to do in order to reduce, or eliminate, the reasons that could cause WMSD. Indeed, for example, only splitting the period of recovery (about $45 \mathrm{~min}$ utes) in four pauses, instead two, the value of the checklist OCRA decrease to 16.51 and 20.63 (red colour) respectively for right and left limbs. 
The cutting operation is not characterized by having actions that are standardized like the industrial cycles. During the cutting operation no movement of loads is required, because the chain saw discharges its weight through the bar in the wood, and also because the penetration of the cutting tooth requires a very low force. However during the relief it was possible to identify an action where the operator moved loads whit repetitive movements: when he picked up the chain saw from the ground and moved it until the trunk and vice versa. Since the chain saw had a weight higher than $3 \mathrm{~kg}$ (chain saw Husqvarna $576 \mathrm{XP}, 6.8 \mathrm{~kg}$ ), since it was moved more than once every 5 minutes and since the operator worked on a floor with poor characteristics of stability, Authors have considered the RNLE approach applicable for the risk assessment due to load movements. For this assessment it was estimated, cautiously due to the short sample period, that the chain saw was moved for 150 times per day of work. In fact the operator did not pick the equipment up from the ground before starting every cutting operation because he had it already in hand. On the other hand, several times the operator interrupted the cutting, put the chain saw on the ground, completed the felling operations through the use of a wedge, than picked the chain saw up again before leaving the place. Often the operator has done this movement while kneeling on the ground. So the geometry and respective factors of multiplication changed. Also this approach, as the checklist OCRA, gives as result a numerical value together with a colour classification. The assessment takes into account the operator's age and gender. Since in the present study-case the operator was a male older than 45 year, the assessed composite lifting index (CLI) is equal to 1.32. This value means a high intrinsic presence of work-correlated risk of injures. Considering the factors that had influenced the LI during the cutting operation, without any doubt, the most important was the horizontal distance. As a matter of fact, during the cutting, he often kept the equipment at a distance higher than $51 \mathrm{~cm}$ in order to ensure a good safety and at the same time to have a good visibility.

The results obtained from the OWAS and REBA assessment are reported in Table 3. In this Table all scores assigned with the two methods for each singular video frame are summarized. The two methodologies assessed different aspects of the posture that the operator assumed during the cutting operation. It is important to underline that the score scales are different for the two methods. In fact the OWAS scale classifies the risk of injures with values from 0 to 400 with intervals every 100 points, meanwhile the REBA with values from 1 to 15 , divided in 5 different intervals. Considering these difference several video frames have substantial difference in the evaluation. For example the scores that the two methodologies assign to frame 5 and 6 are different. For the OWAS the posture in the frame number 5 and 6 is classified as a posture with negligible risk. Meanwhile, for the same posture, REBA assigns a score of high and very high risk of injures. Another difference between the two approaches is that REBA evaluates the posture of both sides of upper limbs. This factor is important to evaluate because the limbs do not always perform the same actions. Difference in the performed actions may cause different risks of
Table 1. Technical actions recognized during the video assessment.

\begin{tabular}{lcc} 
Technical action & $\begin{array}{c}\text { Right hand } \\
\text { count }\end{array}$ & $\begin{array}{c}\text { Left hand } \\
\text { count }\end{array}$ \\
to take & 5 & - \\
to action & 22 & - \\
\hline to place & 3 & 3 \\
to pull/push & 9 & 12 \\
\hline to keep & 1 & 2 \\
to extract & 2 & 2 \\
\hline to throw & 2 & - \\
TOT & 44 & 19 \\
\hline
\end{tabular}

Table 2. Scores attributed for each parameter evaluated.

\begin{tabular}{lcc} 
Parameters of assessment & $\begin{array}{c}\text { Limb } \\
\text { Right }\end{array}$ & left \\
mRP & 1.265 & 1.265 \\
mNRWT & 3.5 & 3.5 \\
\hline Fr & 2.5 & 2.5 \\
F & 4 & 4 \\
\hline Ps & 5.5 & 9.5 \\
Ps (shoulder) & 1 & 2 \\
\hline Ps (elbow) & 1 & 8 \\
Ps (wrist) & 2 & 2 \\
\hline Ps (hand) & 4 & 3 \\
Ps (stereotypy) & 1.5 & 1.5 \\
\hline CF & 4 & 4 \\
checklist OCRA & 19.23 & 24.04 \\
\hline
\end{tabular}

Table 3. Frames every 5 seconds of the video sample with respective OWAS and REBA scoring.

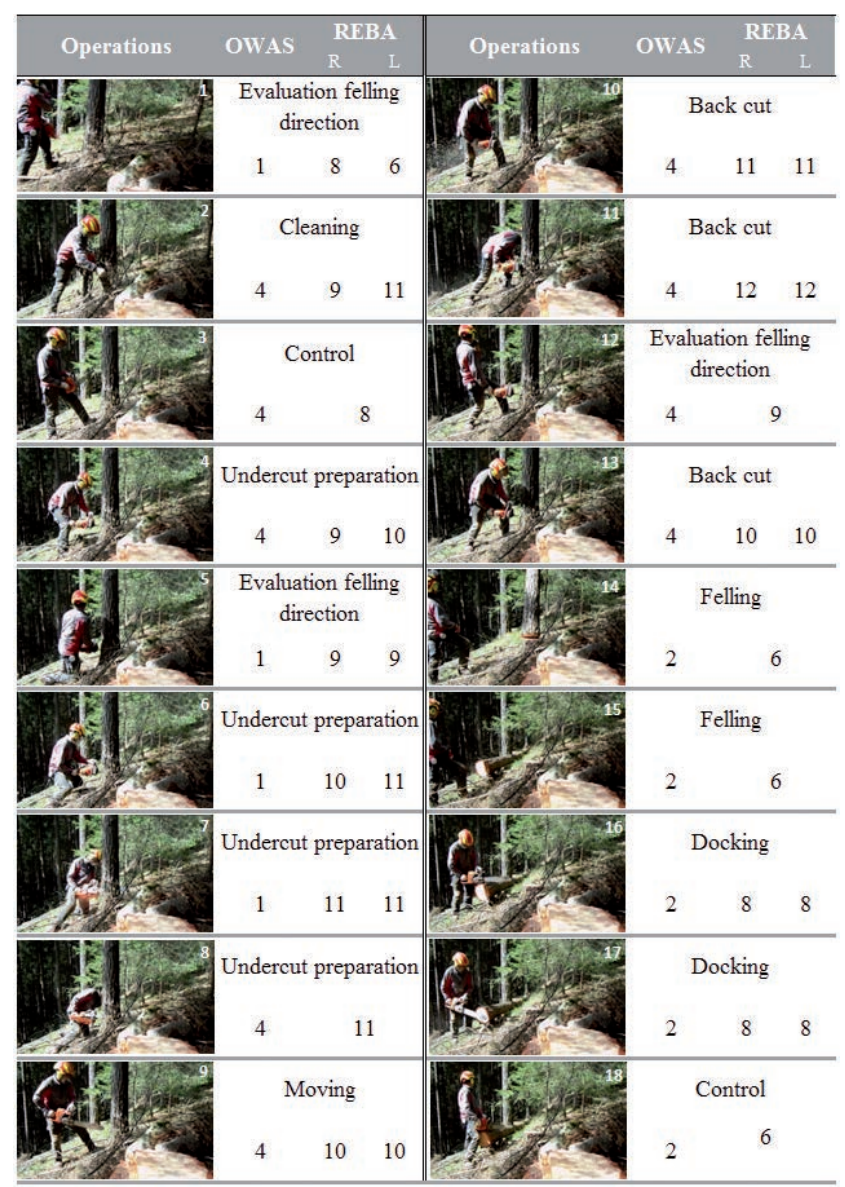


injures.

Results for the OWAS assessment show that the operation of cutting involves a risk of injures equal to 278 . This means that the operator performs a task with a medium risk of injuries.

Results of the REBA assessment show that in several evaluations a difference between the two upper limbs is present. The presence of more than one score for the REBA assessment (Table 3), even if equal, means a difference in the evaluation between the upper limbs postures.

Then, for each side, the percentage of presence was calculated. Since the approach does not foresee the calculation of a global index of risk the same procedure developed for the OWAS assessment was applied. So the global index of risk for cutting task equals to 406 and 411 for right and left upper limbs, recognising that the left upper limb could be more likely affected by risk of injures. Anyhow the result, compared to the REBA classification, highlights that the assessed operation presents a very high level of risk of injures and that it requires immediate action to reduce it.

It is important to underline that the specific literature regarding the methodology for the assessment of WMSD in forestry sector is very poor. In fact only two documents have been usable for a comparison, both only for the OWAS approach (Calvo, 2009; Zanuttini, 2005). From what reported in literature the index of risk results equal to 287 and 156 (Calvo 2009, Zanuttini 2005). Meanwhile the value obtained from this study is 278; in line with those reported in literature for the same operation and in analogues environment of work. So it is possible to draw the conclusion that the cutting phases presents a risk of WMSD ranged between slight and medium risk. The results are affected by the terrain morphology, which influence the working posture, and by the working habitudes.

\section{Conclusions}

The present work is an overview on the application of these different approaches for the assessment of the risk of injures in the primary forestry sector. The approaches applied were:

- OCRA checklist index, for the assessment of the level of risk of injures at upper limbs due to at repetitive movements;

- RNLE index, for the assessment of the level of risk of injures at rachides due to lifting movements;

- OWAS and REBA methods, for the assessment of the presence of risk of injures due to an incorrect posture assumed during the work.

Considering the four approaches applied, results showed that the cutting operation involves a substantial risk of injures, even if with different levels. All approaches presented a good feasibility in their application, thanks to the presence of specific software and also to the easy evaluations required. Considering the four different approaches, only the one related to RNLE is affected by uncertainties on its application due to the short period of sample. This happens because it is not clear if the movements of the chain saw respect the frequency of 1 every 5 minutes. Besides this it is necessary to validate the equation employed to assess the global index of risk for the REBA approach. Indeed the absence of a method to calculate the index for the entire task is a point of weakness of REBA approach. The comparison between the OWAS and REBA approaches showed that REBA has a higher detail level of assessment. Indeed this assessment is based on the measurement of the angles between the body structures. Beside this, it also analyses the body extremities as the wrist, the neck, the elbow and the shoulders for both sides; parameters that are not considered by OWAS approach. Besides these the REBA approach assesses also the type of handle cou- pling and the characteristics of the performed activities. Obviously due to these additional requirements the REBA approach takes more time for the assessment than the OWAS approach. The applications of these approaches have presented also the capability to obtain information on the posture, or on the action, that presents an intrinsic high risk of WMSD. So it is suitable for suggesting the intervention to be performed in order to decrease or delete the source of risk.

However, these approaches were not developed specifically for forestry operations, indeed several times they presented weakness points during the assessment. For example REBA approach does not assign any score when the operator has a kneeling posture. On the other hand, OWAS approach does not consider the posture of the body extremities. Therefore, in order to obtain a more accurate assessment, a review of the assessment parameters as well as the scores, mainly for posture assessment approaches, could be performed.

The position of the camera with respect to the operator, as well as its distance, is a fundamental factor to pay attention to during the filming. As a matter of fact, several videos were not usable because the recording angles, or the high distance from the operator, did not permit a good visibility of the assessment items. In the future, for the assessment of posture and RNLE, the camera will be placed in the front side - the same as the felling direction - and moved on lateral side out of the risk area. Meanwhile for the evaluation of OCRA checklist index the possibility to install a wide angle camera on the operator - on the helmet, or on a specific support clipped on the vest - directed to the hands will be evaluated. As consequence of the good results and in order to perform a global operative monitoring of the entire mechanised forest chain, this experience will be tested also for other forestry operations with the applications of sensors able to assess other ergonomic parameters like noise, vibration, etc.

In conclusion the use of OCRA checklist index, RNLE index, REBA index and OWAS index have presented a good applicability for the assessment of WMSD in the forestry sector, mainly to assess the cutting operations. Anyhow the research will assess also the other forest activities in order to evaluate if these approaches may be used for the analysis of all logging operations. The possibility to apply these assessment methods on the primary forestry sector, together with other ergonomic assessment methods, will permit the evaluation of the forestry firms in other to obtain quality certifications.

\section{References}

Ashby L., Bentley T., Parker R. 2001. Musculoskeletal disorder in silviculture and logging 1995-1999. Center Of Human Factors and Ergonomics (COHFE) Report. Volume 2(3).

Calvo A. 2009. Musculoskeletal Disorder (MSD) risk in forestry. A case Study to suggest an ergonomic analysis. Agricultural Engineering International: the CIGR Ejournal. Manuscript MES 1149, Vol. XI April.

Camilleri D., Caruso L., Schillaci G. 2012. Literature review: application of the OCRA method in agriculture and agro food activities. In: International Conference RAGUSA SHWA 2012 September 3-5. 2012 Ragusa - Italy "Safety Health and Welfare in Agro-food Agricultural and Forest Systems".

Cecchini, M., Massantini, R., Monarca, D. 2010. The ergonomics analysis tools for the assessment of the risk of musculoskeletal disorders due to the repetitive movements of the upper limbs of the workers employed in agriculture. In: "International Conference on Agricultural Engineering-AgEng 2010: towards environmental technologies, Clermont-Ferrand, France, 6-8 September 2010".

Cividini R. 1991. Elementi di tecnologia forestale. Materiale legno, 
prodotti boschivi, mezzi e tecniche della raccolta, lavorazione dei prodotti boschivi. EdAgricole, Bologna. ISBN 88-206-2157-6.

Colantoni A., Marucci A., Monarca D., Pagniello B., Cecchini M., Bedini R. 2012. The risk of musculoskeletal disorders due to repetitive movements of upper limbs for workers employed to vegetable grafting. Journal of Food, Agriculture \& Environment. Vol. 10 (3\&4), July-October 2012.

Colombini D., Occhipinti E. 2011. La valutazione del rischio da sovraccarico biomeccanico degli arti superiori con strumenti semplificati: la minicheklist OCRA. Contenuti, campo applicativo e validazione. Med Lav 2011; 102, 1 000-000.

Colombini D., Occhipinti E., Cerbai M., Battevi N., Placci M. 2011. Aggiornamento di procedure e di criteri di applicazione della Checklist OCRA. Med Lav 2011; 102, 1 000-000.

Colombini D., Occhipinti E., Battevi N., Cerbai M., Fanti M., Menoni 0., Placci M., 2010 - Movimentazione manuale dei carici. Manuale operativo dep l'applicazione del decreto legislativo 81/08. Dossier Ambiente $n^{\circ}$ 89. Ed., Associazione Ambiente e Lavoro. ISSN 18255396

Colombini D., Occhipinti E., Hernandez A., Alvarez, E. Montomoli L., Cerbai M., Fanti M., Ardissone S., Ruschioni A., Giambartolomei M., Sartorelli P. 2007. Repetitive movements of upper limbs in agriculture: set up of annual exposure level assessment models starting from OCRA checklist via simple and practical tools. In: International Conference on Agriculture Ergonomics in Developing Countries, AEDeC 2007.

Hignett, S., McAtamney, L. 2000. Rapid entire body assessment (REBA). Appl. Ergon. 31 (2). pp 201-05.

Hippoliti G., Piegai F. 2000. La raccolta del legno; tecniche e sistemi di lavoro. Compagnia delle Foreste-Arezzo. pp 157

Li G., Buckle P. 1999. Current techniques for assessing physical exposure to work-related musculoskeletal risks, with emphasis on posture-based methods, Ergonomics, 42:5. pp 674-95.

Louhevaara V, Suurnäkki T. 1992. OWAS: a method for the evaluation of postural load during work. Institute of Occupational Health Centre for Occupational Safety, Helsinky. pp 23.
Lundqvist P., Gustafsson B. 1987. Working postures in Dairy barns. IXth Joint International Ergonomics Symposium "Working postures in Agricolture and Forestry”. Kuopio, Finland.

McAtamney L., Hignett S. 2005. Rapid Entire Body Assessment. In: Stanton N., Hedge A., Brookhuis K., Salas E., Hendrick H., "Handbook of Human Factors and Ergonomics Methods". CRC Press LLC. ISBN 0-415-28700-6.

Murgia l., Rosecrance J.C., Gallu T., Paulsen R. 2012. Risk evaluation of upper extremity musculoskeletal disorders among cheese processing workers: A comparison of exposure assessment techniques. In: International Conference RAGUSA SHWA 2012 September 3-5. 2012 Ragusa - Italy "Safety Health and Welfare in Agro-food Agricultural and Forest Systems".

Niosh, 1981. Work practices guide for manual lifting. Niosh Technical Report. Publication $n^{\circ} 81-122$. US Department of Health and Human Service 1981.

Pressiani, S., \& Colombini, D. 2010. Risk Assessment in plant nursery characterized by several working task with annual turnover. In International Conference Ragusa SHWA2010 "Work Safety and Risk Prevention in Agro-food and Forest Systems".

Schillaci G., Caruso L., Balloni S., Camilleri D 2011. I rischi da sovraccarico biomeccanico nelle attivitá del vivaio orticolo. In: Convegno di Medio Termine dell'Associazione Italiana di Ingegneria Agraria Belgirate, 22-24 settembre 2011.

Waters T.R., Lu M.L., Occhipinti E. 2007. New procedure for assessing sequential manual lifting jobs using the revised NIOSH lifting equation. Ergonomics. 50(11): 1761-1770.

Waters T.R., Putz-Anderson V., Garg A. 1994. Applications Manual for the Revised NIOSH Lifting Equation. DHHS(NIOSH) Publication No. 94-110. National Institute for Occupational Safety and Health, Centers for Disease Control and Prevention. Cincinnati, Ohio. 45226.

Zanuttini R., Cielo P., Poncino D. 2005. Il metodo OWAS. Prime applicazioni nella valutazione del rischio di patologie muscolo-scheletriche nel settore forestale in Italia. Forest@ 2(2). 242-55. [online] URL: http://www.sisef.it/ 